Itinéraires Itinéraires

Littérature, textes, cultures

2012-2 | 2012

Intime et politique

\title{
Simone de Beauvoir, du Deuxième sexe à $L a$ Vieillesse : quand l'intime gagne le politique
}

Anne Strasser

\section{(2) OpenEdition}

\section{Journals}

Édition électronique

URL : http://journals.openedition.org/itineraires/1135

DOI : 10.4000/itineraires. 1135

ISSN : 2427-920X

Éditeur

Pléiade

\section{Édition imprimée}

Date de publication : 1 novembre 2012

Pagination : 93-103

ISBN : 978-2-336-00027-5

ISSN : $2100-1340$

\section{Référence électronique}

Anne Strasser, «Simone de Beauvoir, du Deuxième sexe à La Vieillesse : quand l'intime gagne le politique », Itinéraires [En ligne], 2012-2 | 2012, mis en ligne le 01 novembre 2012, consulté le 10 décembre 2020. URL : http://journals.openedition.org/itineraires/1135 ; DOI : https://doi.org/10.4000/ itineraires. 1135

\section{(c) $($ ) $(9)$}

Itinéraires est mis à disposition selon les termes de la licence Creative Commons Attribution - Pas d'Utilisation Commerciale - Pas de Modification 4.0 International. 


\title{
Simone de Beauvoir, du Deuxième sexe à La Vieillesse : quand l'intime gagne le politique
}

\begin{abstract}
Philosophical essays, Le Deuxième Sexe (1949) and La Vieillesse (1970) have a political impact. Yet, even if there are just hints of private life in the first essay, Beauvoir explicitely confides her own experience in the second one. Between 1949 and 1970, the writer's situation, as well as her writing evolved: she wrote four autobiographies. The second essay is close to an autobiography with a narrow intertextuality revealing a constant dialectic between her unique private experience and the universal and political impact she wants to confer on her work.
\end{abstract}

Keywords : Simone de Beauvoir, private experience, politics, autobiography, essay Mots clés : Simone de Beauvoir, expérience privée, politique, autobiographie, essai

Simone de Beauvoir, figure de l'intellectuelle engagée, a vu souvent ses œuvres romanesques et autobiographiques questionnées à la lueur de sa pensée philosophique, notamment de son analyse de la condition des femmes. De même, ses autobiographies consacrent de longs passages à la vie politique, à tel point qu'elles relèvent davantage des Mémoires que du genre autobiographique proprement dit. Moins évidente est la recherche de l'écrivaine et de la femme dans ses essais philosophiques, qu'on peut qualifier de politiques ici, dans le sens où ils visent, par une approche philosophique, à modifier en profondeur les représentations sociales. Or, il est remarquable que c'est alors qu'elle s'apprête à écrire sur elle-même, en 1947, que Simone de Beauvoir entreprend l'écriture du Deuxième Sexe ${ }^{1}$. Ainsi explique-t-elle dans La Force des choses :

Je sentais le besoin d'écrire au bout de mes doigts, et le goût des mots dans ma gorge, mais je ne savais qu'entreprendre. [...] En fait, j'avais envie

1. Simone de Beauvoir, Le Deuxième Sexe I et II [1949], Paris, Gallimard, coll. "Folio Essais », 1986. 
de parler de moi. [...] Je m'avisai qu'une première question se posait : qu'est-ce que ça avait signifié pour moi d'être une femme? [...] Je fus si intéressée que j'abandonnai le projet d'une confession personnelle pour m'occuper de la condition féminine dans sa généralité ${ }^{2}$.

De même, c'est en partie à la suite des réactions suscitées par son récit autobiographique La Force des choses qu'elle a décidé d'écrire La Vieillesse :

Dans la France d'aujourd'hui, [la vieillesse] est aussi un sujet interdit. Quand à la fin de La Force des choses j'ai enfreint ce tabou, quel tollé j'ai soulevé! [...] Avec gentillesse ou colère un grand nombre de gens, surtout de gens âgés, m'ont abondamment répété que la vieillesse, ça n'existe pas ! [...] Voilà pourquoi j'écris ce livre : pour briser la conspiration du silence ${ }^{3}$.

Cependant, si d'une certaine manière la démarche autobiographique a été l'origine, voire le moteur de l'écriture de ces deux essais, ils sont très différents quant à la part autobiographique, la part intime pourrait-on dire, qu'ils comportent. Nous prendrons ici le mot intime à la fois dans le sens de « privé » ou de " personnel » par opposition à ce qui est « public » et visible, et dans son sens étymologique, à savoir ce qui est intérieur, et même le plus intérieur, conformément au superlatif latin dont est issu l'adjectif « intime ». En effet, si l'expérience personnelle affleure dans Le Deuxième Sexe, elle n'est jamais présentée comme telle, alors que La Vieillesse est nourrie de la propre expérience de Beauvoir, racontée par un « je » assumé. Cette différence très nette mérite d'être étudiée à la lueur de la situation de l'auteure au moment de la publication de ces deux livres parus respectivement en 1949 et 1970, mais aussi de façon plus inédite à la lueur des pratiques d'écriture de Simone de Beauvoir. En effet, l'écriture autobiographique à laquelle elle s'est livrée entre la parution de ces deux essais éclaire également leurs différences, tant parce qu'elle informe le contenu que parce qu'elle prend en compte les lecteurs.

Ainsi, après avoir expliqué l'évolution de la place de l'intime dans ces deux ouvrages, nous étudierons spécifiquement la présence de l'intime dans $\mathrm{La}$ Vieillesse avant de montrer que ce dernier essai est nourri d'une intertextualité étroite avec l'autobiographie, intertextualité qui révèle combien chez Beauvoir l'écriture de l'intime et l'analyse philosophique et politique se rejoignent.

\section{De l'analyse distante à l'expérience vécue}

Beauvoir elle-même a souhaité que dans son œuvre, ces deux essais se fassent écho. Quand elle revient dans Tout compte fait sur l'écriture de La Vieillesse, elle explique :

2. Simone de Beauvoir, La Force des choses $I$ [1963], Paris, Gallimard, coll. «Folio », 1972, p. 135-136., p. 135-136.

3. Simone de Beauvoir, La Vieillesse I [1970], Paris, Gallimard, coll. « Idées », 1979, p. 9-10. 
En mai 67, j'avais terminé les trois récits réunis sous le titre La Femme rompue ${ }^{4}$. Je me demandais ce que j'allais faire. Presque tout de suite j'ai eu une illumination : cette question que $\mathrm{j}$ 'avais échoué à traiter sous une forme romanesque, la vieillesse, je l'étudierais dans un essai qui serait, touchant les personnes âgées, le symétrique du Deuxième Sexe. [...] Dans ce cas aussi c'est d'abord l'idée d'une démystification qui m'a séduite. Mais si je me suis décidée c'est que j'éprouve le besoin de connaître dans sa généralité la condition qui est la mienne. Femme, j'ai voulu élucider ce qu'est la condition féminine; aux approches de la vieillesse, j'ai eu envie de savoir comment se définit la condition des vieillards ${ }^{5}$.

La méthode utilisée est identique et elle repose sur la philosophie existentialiste. Dans un premier temps, elle étudie la situation des vieillards en extériorité - telle qu'elle apparaît à autrui - et dans un second temps en intériorité : à savoir comment l'homme âgé « intériorise son rapport au corps, au temps, à autrui ${ }^{6} \gg$. L'analyse est similaire : la situation des vieillards est étroitement liée à la place que la société leur assigne, ou plutôt ne leur assigne pas. $\mathrm{Au}$ « on ne naît pas femme, on le devient », répond « [la vieillesse] n'est pas seulement un fait biologique, mais un fait culturel ${ }^{7} \gg$.

Cependant, la situation de Beauvoir, au sens existentialiste du terme, est différente au moment de l'écriture des deux essais. Or, comme elle l'explique dans le prologue de La Force de l'âge: « un livre ne prend son vrai sens que si l'on sait dans quelle situation, dans quelle perspective et par qui il a été écrit ${ }^{8} »$. Certes, l'auteure est une femme, cependant elle estime n'avoir pas subi le sort de la plupart des femmes; c'est donc avec une certaine distance qu'elle se lance dans son étude de la condition féminine :

\footnotetext{
j'attachais peu d'importance aux conditions réelles de ma vie : rien n'entravait, croyais-je, ma volonté. Je ne niais pas ma féminité, je ne l'assumais pas non plus : je n'y pensais même pas. J'avais les mêmes libertés et les mêmes responsabilités que les hommes. La malédiction qui pèse sur la plupart des femmes, la dépendance, me fut épargnée ${ }^{9}$.
}

En revanche, elle revendique clairement la dimension personnelle de La Vieillesse, notamment pour la deuxième partie : « Ce qu'il y a d'essentiel dans un travail de cette espèce, ce sont les questions que l'auteur se pose; seules ma propre expérience et ma réflexion m’ont amenée à définir

4. Simone de Beauvoir, La Femme rompue [1967], Paris, Gallimard, 1968. Repris avec Monologue et L'Âge de discrétion, coll. « Folio », 1972.

5. Simone de Beauvoir, Tout compte fait [1972], Paris, Gallimard, coll. «Folio », 1978, p. 183.

6. Simone de Beauvoir, La Vieillesse I, op. cit., p. 21.

7. Ibid., p. 26.

8. Simone de Beauvoir, La Force de l'âge [1960], Paris, Gallimard, coll. « Folio », 1986, p. 12.

9. Ibid., p. 418. 
celles que j'ai traitées $[\ldots]^{10} \gg$. Ainsi, son expérience de la vieillesse nourrira son essai, plus que ne l'a fait son expérience de femme.

La différence tient également à la conscience toute particulière que Beauvoir a du temps. On peut parler d'une conscience traumatique du temps : la jeune Beauvoir était déjà effrayée de grandir par crainte de devenir autre ${ }^{11}$. À moins de trente ans (elle a vingt-six ans en 1934), elle se sent vieille : «J'avais un autre souci : je vieillissais. Ni ma santé ni mon visage n'en pâtissaient; mais de temps en temps, je me plaignais qu'autour de moi tout se décolorât : je ne sens plus rien, gémissais-je $\mathrm{e}^{12}$ ».

Cette différence de situation a une incidence sur l'écriture. Ainsi on ne décèle dans Le Deuxième Sexe que des traces d'intime. Comme l'analyse Éliane Lecarme-Tabone, «né d'un désir d'autobiographie, Le Deuxième Sexe en garde profondément, quoique secrètement, les traces ${ }^{13} »$. En effet, si le « je » est employé, ce n'est pas un « je » qui renvoie à une expérience directe de la condition féminine. Il s'agit du « je » témoin. Ce n'est en fait que rétrospectivement, une fois l'autobiographie publiée, qu'on peut réellement identifier ces traces de l'intime. En effet, on pourra rapprocher telle analyse de la « jeune fille » de la figure de Zaza, de même qu'on découvrira à la lecture des Mémoires d'une jeune fille rangée que la « jeune Simone a, elle aussi, échafaudé des théories baroques sur le mariage et la procréation. [Qu'] elle a vécu ses premières règles dans la honte et dans l'effroi... ${ }^{14}$ ». De même, la figure de la mère dans Le Deuxième Sexe emprunte quelques traits à la mère de l'auteure. Enfin, la publication de la correspondance avec Nelson Algren permettra « d'identifier, derrière la relation amoureuse réussie décrite dans Le Deuxième Sexe, celle que Simone de Beauvoir découvrit avec son amant américain ${ }^{15} »$. Ces «traces » de l'intime sont présentes, mais ne sont perceptibles que dans un espace autobiographique construit au fur et à mesure de l'écriture de l'œuvre. Et ce qui nous importe ici, c'est qu'elles ne sont pas repérables pour le lecteur de $1949^{16}$.

10. Simone de Beauvoir, Tout compte fait, op. cit., p. 185.

11. Il est remarquable, que le 17 avril 1927, à 19 ans, elle écrive déjà dans son cahier : « J'aimerais écrire un roman : d'une jeune fille que son futur visage de 40 ans effraie tant qu'elle emploie toutes ses forces à se refuser à vivre. » Cahiers de jeunesse 1926-1930, texte établi, édité et présenté par Sylvie Le Bon de Beauvoir, Paris, Gallimard, 2008, p. 307. 12. Simone de Beauvoir, La Force de l'âge, op. cit., p. 239.

13. Éliane Lecarme-Tabone, Le Deuxième Sexe de Simone de Beauvoir, Paris, Gallimard, coll. « Foliothèque », 2008, p. 187.

14. Ibid., p. 192.

15. Ibid., p. 192-193. Eliane Lecarme-Tabone a approfondi cette étude dans « Essai et autobiographie : du Deuxième Sexe aux Mémoires d'une jeune fille rangée », Les Temps modernes, $\mathrm{n}^{\circ} 654,2009$, p. .

16. Ce qui est paradoxal, et mériterait d'être étudié par ailleurs, est que malgré cette absence d'implication personnelle, la parution du Deuxième Sexe a donné lieu à une débauche d'injures toutes personnelles (voir notamment Simone de Beauvoir, La Force des choses I, op. cit., p. 260). 
Un autre élément de la situation de l'auteure vient éclairer cette différence de posture d'écriture entre Le Deuxième Sexe et La Vieillesse. Entre 1949 et 1970, elle a écrit quatre récits autobiographiques, Les Mémoires d'une jeune fille rangée (1958), La Force de l'âge (1960), La Force des choses (1963) et Une Mort très douce (1964). Le désir d'autobiographie qui a présidé à l'écriture de son premier essai s'est concrétisé et elle a osé parler d'elle. À ce que ses contemporains pouvaient connaître d'elle en tant que figure publique de la vie intellectuelle, ce qu'elle est dès avant 1949, est venue s'ajouter une connaissance plus intime à travers ses écrits autobiographiques. Ainsi ce qu'elle peut évoquer d'intime dans La Vieillesse, n'est plus " privé », ni secret, elle peut en parler avec d'autant plus de liberté qu'elle l'a déjà fait. Plus intéressant est ce qui se joue du côté du lecteur. En effet, le lecteur de La Vieillesse peut avoir lu ces œuvres, et il est remarquable que Beauvoir s'adresse très clairement à ses lecteurs dans cet essai. Dès les premières pages, elle les interpelle : «c'est pourquoi il est nécessaire de briser le silence : je demande à mes lecteurs de m'y aider ${ }^{17} »$. On note qu'elle n'écrit pas " aux lecteurs », mais à " mes lecteurs », sousentendant par l'adjectif possessif non seulement ceux qui lisent cet essai mais aussi ceux qui l'ont lue auparavant.

Ainsi, la situation de Simone de Beauvoir comme ses conditions d'écriture sont assez différentes en 1947 et 1970. Comment cet intime, à l'état de traces rétrospectives dans Le Deuxième Sexe, s'exprime-t-il dans La Vieillesse?

\section{La Vieillesse : intime et politique à travers les usages du « je "}

On peut aborder cette présence de l'intime par l'intermédiaire du « je ». Le pronom personnel de la première personne du singulier renvoie grammaticalement à la personne qui écrit ou qui parle. Or, l'emploi du « je » dans cet essai s'avère moins simple que cette définition et ses différents usages dessinent des frontières perméables entre l'intime et le politique.

Le « je » renvoie avant tout à Beauvoir en tant qu' elle écrit cet essai, et donc à la situation d'énonciation. Ce « je » est présent quand elle commente ce qu'elle écrit ou explique sa méthode : « Voilà justement pourquoi j'écris ce livre : pour briser la conspiration du silence ${ }^{18}$ », ou encore « Dans les pages suivantes, je me limiterai à étudier les sociétés occidentales ${ }^{19} »$. À ce « je » peut se substituer le « nous » de majesté, propre aux écrits théoriques et équivalent dans son usage : "nous l' [l'homme âgé] avons décrit en extériorité. Il est aussi un sujet qui intériorise sa situation et qui y réagit ${ }^{20} »$. Le « je » se fait également témoin, référence à celle qui écrit et qui a vu :

17. Simone de Beauvoir, La Vieillesse I, op. cit., p. 18.

18. Ibid., p. 10.

19. Ibid., p. 145.

20. Simone de Beauvoir, La Vieillesse II [1970], Paris, Gallimard, coll. « Folio », 1979, p. 11. 
« j'ai visité un hospice de l'Assistance publique, très bien situé, en plein Paris. [...] j'ai vu des êtres humains réduits à une totale abjection ${ }^{21} \gg$. Ce « je » a pour référent Simone de Beauvoir mais n'ouvre pas à l'intime.

C'est le cas d'un autre usage du «je» que nous nommerons le « je philosophique » : usage bien particulier où le pronom renvoie au sujet en tant qu'être pensant, et non à la personne. Il est attendu que dans cet essai, s'appuyant pour une grande part sur une approche philosophique, ce « je » soit couramment utilisé. Ainsi, reprenant la notion d' «irréalisable» définie par Sartre et qui peut recouvrir la vieillesse mais aussi la mort, Simone de Beauvoir écrit : « le pour-soi ne peut ni l' [la mort] atteindre ni se projeter vers elle; elle est la limite externe de mes possibilités et non ma propre possibilité ${ }^{22} »$. Ce « je » philosophique a une portée large puisqu'il vise tout sujet, et non seulement la personne qui s'exprime. Cependant ce « je » philosophique glisse vers un « je » que nous pourrions nommer « intime » et par lequel Beauvoir fait référence à son expérience vécue : ainsi dans ce passage cité, le glissement s'opère dans un premier temps par l'accord au féminin entraîné par l'usage du « je »: « Je serai morte pour les autres, non pour moi : c'est l'autre qui est mortel dans mon être. Je me connais mortelle - comme je me connais vieille - en prenant le point de vue des autres sur moi $^{23} »$. L'usage du féminin est inévitable grammaticalement, mais il teinte de connotations personnelles l'usage de ce « je » qui, employé par un philosophe, serait resté soit masculin, soit neutre. Ici, la dimension « politique » du « je »- politique au sens où ce « je » sert à dénoncer la condition des vieillards - universel se double d'une dimension personnelle, et c'est l'ambivalence de l'emploi de ce « je » par une femme qui crée cette double dimension.

De même la première personne du pluriel est fréquemment employée pour désigner « je » et les autres. À la différence de ce qu'elle fait dans son premier essai, Beauvoir s'associe là aux personnes âgées qu'elle décrit : «L'âge fond sur nous par surprise et nous en éprouvons un obscur sentiment d'injustice ${ }^{24} »$. Ce «nous » rejoint dans son usage le « je » philosophique, en ce sens que ces deux pronoms renvoient à celle qui parle en l'associant aux autres. Cependant la première personne du pluriel glisse plus facilement $\mathrm{au}$ « je » personnel, à savoir un « je » qui renvoie à Beauvoir en particulier, et non à Beauvoir en tant qu'individu parmi d'autres. Ainsi, évoquant les images du passé, l'auteure écrit :

Elles prennent souvent un caractère de clichés : nous les évoquons sans les modifier, sans les enrichir, puisque nous ne saurions découvrir en elles que ce que nous y avons mis. Souvent j'amalgame en un seul souvenir des

21. Simone de Beauvoir, La Vieillesse I, op. cit., p. 412.

22. Simone de Beauvoir, La Vieillesse II, op. cit., 248.

23. Ibid., p. 248-249.

24. Ibid., p. 302. 
données appartenant à différentes époques : à travers toute mon enfance, les visages de Louise, de mon père, de mon grand-père sont immuables ${ }^{25}$.

$\mathrm{Ce}$ « je » qui renvoie à la personne de Beauvoir est très présent. Il lui permet de décrire sa propre perception de la vieillesse : « À vingt ans, à quarante ans, me penser vieille, c'est me penser autre. Il y a quelque chose d'effrayant dans toute métamorphose. J'étais stupéfaite, enfant, et même angoissée quand je réalisais qu'un jour je me changerais en grande personne ${ }^{26} »$; ou encore d'évoquer sa propre expérience de la mort des amis chers comme Dullin, Camus, Merleau-Ponty, Giacometti : « Tant qu'ils vivaient, il n'était pas besoin de souvenir pour qu'en eux notre commun passé demeurât vivant ${ }^{27} »$.

Parlant d'elle-même, elle évoque naturellement son activité d'écriture et notamment d'écriture autobiographique. L'écrivain, mieux qu'un autre, peut lutter à sa manière contre le temps qui passe. Beauvoir montre qu'à chaque fois qu'un être cher disparaît, c'est une partie du passé qui est englouti avec lui : « La mort d'un proche, d'un ami, ne nous prive pas seulement d'une présence mais de toute cette partie de notre vie qui est engagée avec eux ${ }^{28}$. 》 « Dans quelle mesure la mémoire permet-elle de récupérer nos $\operatorname{vies}^{29}$ ? » Si la mémoire est imparfaite, elle permet cependant, soutenue par l'activité d'écriture, de « sauver » ce passé et de le ressusciter dans les pages.

Le « je » se révèle donc multiple : référence simple à la situation d'énonciation, il est également à la fois un «je» intime, nourri de la manière dont Beauvoir a justement « intériorisé » sa vieillesse, et un « je » philosophique, sujet parmi d'autres sujets, comme un « je » parmi les autres quand il est englobé dans le «nous », ces deux derniers emplois prenant une dimension « politique » dans la mesure où ils dépassent le seul individu qu'est Beauvoir et servent la visée politique de cet essai, à savoir contribuer à modifier les représentations de la vieillesse.

Cependant, quand Beauvoir évoque sa propre expérience, le lecteur est sensible à une forme d'écho entre ses œuvres. Ce « je » intime est peut-être plus précisément un « je » autobiographe, dans la mesure où l'expérience vécue personnellement dont elle fait part dans La Vieillesse est souvent une expérience qu'elle a déjà racontée, d'où une intertextualité marquée.

\section{L'intertextualité ou la conjonction de l'intime et du politique}

La Vieillesse, présenté comme une réflexion théorique, philosophique et générale sur la vieillesse, est aussi et peut-être avant tout, une réponse

25. Ibid., p. 136.

26. Simone de Beauvoir, La Vieillesse I, op. cit., p. 14-15.

27. Simone de Beauvoir, La Vieillesse II, op. cit., p. 140.

28. Ibid., p. 139.

29. Ibid., p. 133. 
aux critiques qui ont accueilli La Force des choses. En effet, Beauvoir avait conclu ce récit autobiographique par une expression profondément désenchantée :

Je revois la haie de noisetiers que le vent bousculait et les promesses dont j'affolais mon cœur quand je contemplais cette mine d'or à mes pieds, toute une vie à vivre. Elles ont été tenues. Cependant, tournant un regard incrédule vers cette crédule adolescente, je mesure avec stupeur à quel point j'ai été flouée ${ }^{30}$.

Dans La Vieillesse, elle va s'attacher à montrer comment on peut être amené à exprimer ce désenchantement et donc à provoquer un malentendu. Ainsi elle s'attaque à un préjugé : «Avoir réussi sa vie, cela ne peut-il pas suffire à combler un individu déclinant? C'est ce qu'on imagine quand on est jeune ${ }^{31} »$. Mais c'est une erreur : «Un grand malentendu sépare les gens qui regardent du dehors un homme "arrivé", dans l'apparente plénitude de son être-pour-autrui, et l'expérience vécue qu'il a de lui-même ${ }^{32}$. » Elle explique cet écart philosophiquement :

Sartre a expliqué ce décalage dans L'Être et le Néant : « Le futur ne se laisse pas rejoindre, il glisse au passé comme ancien futur... [...] Même si mon présent est rigoureusement identique par son contenu au futur vers quoi je me projetais par-delà l'être, ce n'est pas ce présent vers quoi je me projetais car je me projetais vers ce futur en tant que futur, c'est-à-dire en tant que point de rejoignement de mon être. »

Et elle enchaîne :

C'est pourquoi j'ai pu sans contradiction écrire dans les Mémoires d'une jeune fille rangée: "Aucune vie, aucun instant d'aucune vie ne saurait tenir les promesses dont j'affolais mon cœur crédule »; et dans La Force des choses : "Les promesses ont été tenues », tout en concluant: «J'ai été flouée. » Le présent, même conforme à mes attentes, ne pouvait pas m'apporter ce que j'attendais : la plénitude d'être à quoi tend vainement l'existence. Le Pour-soi n'est pas. Et nul ne peut dire : «J'ai eu une belle vie » parce qu'une vie on ne l'a pas $^{33}$. »

De même, elle explique qu'en écrivant La Force des choses, elle était encore victime de « fétichismes et de mirages », dont la vieillesse libère : « j'ai accepté depuis longtemps l'idée que pour 1'existant la recherche de l'être est vaine : jamais le pour-soi ne se réalisera comme en soi. J'aurais

30. Simone de Beauvoir, La Force des choses II [1963], Paris, Gallimard, coll. « Folio », 1972, p. 508.

31. Simone de Beauvoir, La Vieillesse II, op. cit., p. 140.

32. Ibid., p. 141.

33. Ibid., p. 141-142. 
dû prendre mon parti de ce fatal échec et ne pas rêver cet absolu dont j'ai déploré l'absence, à la fin de La Force des choses ${ }^{34} \gg$.

Au-delà de cette référence à La Force des choses, l'essai est empreint d'autobiographie et c'est peut-être là la forme la plus originale de la présence de l'intime.

Beauvoir y évoque en effet des événements personnels qu'elle a déjà racontés. Ainsi reprendra-t-elle, pour illustrer les intermittences de la mémoire, un épisode important de son amitié avec Zaza, épisode raconté dans les Mémoires d'une jeune fille rangée $e^{35}$ : « Âgée de 12 ans, Zaza dans la salle d'étude des cours me remercie d'un sac dont je lui fais cadeau : elle a la silhouette et les traits de ses 20 ans $^{36}$. » De même, lorsque dans La Vieillesse, elle évoque la mort d'amis chers, certaines expressions renvoient explicitement à des passages de l'autobiographie : « je me rappelle mon désarroi à la mort de Dullin, avec qui je n'avais pourtant pas eu de vraie intimité. C'était tout un pan de ma propre vie qui s'effondrait : Ferroles, l'Atelier, les répétitions des Mouches, les dîners si gais où il racontait ses souvenirs, évanouis avec lui ${ }^{37} »$. Or, elle écrit dans La Force des choses à propos de la mort de Dullin : "Tout un pan de mon passé s'effondrait et j'eus l'impression que ma propre mort commençait ${ }^{38}$. »

Comme on le voit dans ce dernier exemple, au-delà de l'événement lui-même, déjà raconté auparavant, les mêmes mots sont souvent repris de l'autobiographie à l'essai, reprise très perceptible pour le lecteur. Ainsi lit-on dans La Vieillesse : « La vieillesse n'est pas réservée aux autres; elle nous guette ${ }^{39}$ », ou encore : « Déjà 40 ans, je suis restée incrédule quand, plantée devant un miroir, je me suis dit: "J'ai 40 ans" 40 ", écho de ces passages de l'autobiographie : «Quarante ans. Quarante et un. Ma vieillesse couvait. Elle me guettait au fond du miroir. Cela me stupéfiait qu'elle marchât vers moi d'un pas si sûr alors qu'en moi rien ne s'accordait avec elle ${ }^{41}$ », ou encore « Pour m'en convaincre, je n'ai qu'à me planter devant la glace. À quarante ans, un jour, j'ai pensé : “Au fond du miroir la vieillesse guette; et c'est fatal, elle m'aura." Elle m'a ${ }^{42}$ ", exact écho de la citation précédente. La nécessité et la difficulté de se convaincre de cette vieillesse qui attaque de l'extérieur sont communes à l'essai et à l'autobiographie.

34. Ibid., p. 325.

35. Simone de Beauvoir, Mémoires d'une jeune fille rangée [1958], Paris, Gallimard, coll. « Folio », 2008, p. 166-167.

36. Simone de Beauvoir, La Vieillesse II, op. cit., p. 136.

37. Ibid., p. 140.

38. Simone de Beauvoir, La Force des choses I, op. cit., p. 273.

39. Simone de Beauvoir, La Vieillesse I, op. cit., p. 236.

40. Simone de Beauvoir, La Vieillesse II, op. cit., p. 13.

41. Simone de Beauvoir, La Force des choses I, op. cit., p. 234.

42. Simone de Beauvoir, La Force des choses II, op. cit., p. 505. 
De même les lecteurs des Mémoires d'une jeune fille rangée reconnaîtront la jeune Beauvoir: " À quinze ans, feuilletant mes livres de classes neufs, la traversée de l'année scolaire me semblait une grande et passionnante expédition. [...] l'immensité de l'avenir déployé à mes pieds m'exaltait : quarante ans, soixante ans à vivre, c'était l'éternité puisque une année me paraissait si vaste ${ }^{43} 》$. Ceux de La Force des choses entendront les mêmes mots : " Je revois la haie de noisetiers que le vent bousculait et les promesses dont $\mathrm{j}$ 'affolais mon cœur quand je contemplais cette mine d'or à mes pieds, toute une vie à vivre ${ }^{44} »$; la même métaphore de ce vaste avenir - comme l'expriment les termes « déployé », « mine»-plein de promesses - suggérées par les expressions « m'exaltait», « mine d'or »comme offert, puisqu' « à [ses] pieds », est très éloquente.

Enfin, certains passages de La Vieillesse, sans nécessairement faire écho à des passages de l'autobiographie, empruntent cependant le ton, la forme de cette écriture de soi, et pourraient figurer tels quels dans un récit autobiographique. Ainsi, lorsque l'auteure explique que l'idée de la mort l'épouvante moins qu'autrefois : «elle est absence au monde, et c'est de cette absence que je ne pouvais prendre mon parti. Mais tant d'absences se sont déjà creusées en moi! Mon passé est absent, absents les amis morts, les amis perdus, et tant d'endroits où je ne retournerai jamais. Quand l'absence aura tout englouti, cela ne fera pas beaucoup de différence ${ }^{45} »$. La phrase exclamative, la répétition du mot «absence », les termes renvoyant à la perte définitive, sont autant de procédés qui donnent une tonalité très intime et émouvante à l'essai.

L'intertextualité est donc la marque la plus profonde de ce lien dans l'écriture de Beauvoir entre l'intime et le politique. Elle dessine l'espace autobiographique à l'intérieur duquel les œuvres de Beauvoir sont à lire. Dans l'essai se manifeste aussi ce souci constant qu'a eu Beauvoir de communiquer « de la manière la plus directe, le goût de [sa] propre vie ${ }^{46}$ ». L'analyse philosophique comme sa propre expérience visent à servir, à « changer la vie ${ }^{47}$ » de ses contemporains.

Ainsi l'intime, à l'état de traces rétrospectives dans Le Deuxième Sexe, occupe une large place dans La Vieillesse. Différence de « situation »- en tant qu'écrivain, Beauvoir est devenue une écrivaine reconnue, a un lectorat fidèle qui a lu ses autobiographies -, mais aussi différence d'expérience, une conscience aiguë du vieillissement face à une relative distance quant à la condition de la plupart des femmes.

43. Simone de Beauvoir, La Vieillesse II, op. cit., p. 151.

44. Simone de Beauvoir, La Force des choses II, op. cit., p. 508.

45. Simone de Beauvoir, La Vieillesse II, op. cit., p. 253.

46. Simone de Beauvoir, Tout compte fait, op. cit., p. 634.

47. Simone de Beauvoir, La Vieillesse II, op. cit., p. 400. Ce sont les derniers mots de cet essai. 
La présence de l'intime dans La Vieillesse se repère à travers l'usage de la première personne du singulier. Le " je » philosophique et impersonnel au service d'une pensée engagée coexiste avec un « je » intime, qui se révèle un " je » autobiographe en tant qu'il ouvre à une expérience déjà racontée dans l'autobiographie. La coexistence de ces deux « je », et donc de l'intime et du politique, nous semble illustrer l'articulation entre singulier et universel que Beauvoir a très souvent abordée. Pour Beauvoir, « qu'il s'agisse d'un roman, d'une autobiographie, d'un essai, d'un ouvrage d'histoire, de n'importe quoi, l'écrivain cherche à établir une communication avec autrui à partir de la singularité de son expérience vécue : son œuvre doit manifester son existence et porter sa marque; et c'est par son style, son ton, le rythme de son récit qu'il la lui imprime ${ }^{48} »$. Elle distingue l'écrivain et le philosophe :

La philosophie considère l'homme en tant que notion; elle veut connaître son rapport total à l'univers. L'écrivain lui aussi vise l'universel mais à partir de sa singularité. Il ne prétend pas livrer un savoir, mais communiquer ce qui ne peut pas être $s u$ : le sens vécu de son être dans le monde. Il le transmet à travers un universel singulier : son œuvre ${ }^{49}$.

L'écrivain et la philosophe se confondent dans La Vieillesse, l'intime et le politique se rejoignent, l'essai rejoint l'autobiographie confirmant qu'il y a bien chez cette auteure une dialectique constante entre la singularité de son expérience et la portée universelle qu'elle veut lui conférer, soit dans l'écriture de soi, soit dans l'essai.

Ainsi à la raison d'être de l'autobiographie : « Samuel Pepys ou JeanJacques Rousseau, médiocre ou exceptionnel, si un individu s'expose avec sincérité, tout le monde, plus ou moins, se trouve mis en jeu. Impossible de faire la lumière sur sa vie sans éclairer ici ou là, celle des autres ${ }^{50} »$ répond la démarche philosophique :

Le philosophe, c'est celui pour qui l'homme est en question dans son être, c'est celui qui s'interroge sur la condition humaine prise dans sa totalité. Mais il est lui-même un homme, tout l'homme : ce qu'il a à dire, c'est soi-même, dans son universalité ${ }^{51}$.

Anne Strasser

Université de Lorraine, CELJM/LIS (EA 3962)

48. Simone de Beauvoir, Tout compte fait, op. cit., p. 163.

49. Simone de Beauvoir, La Vieillesse II, op. cit., p. 187.

50. Simone de Beauvoir, La Force de l'âge, op. cit., p. 12.

51. Simone de Beauvoir, La Vieillesse II, op. cit., p. 182. 